\title{
CONSTITUIÇÃO DE COMUNIDADES DE PRÁTICA NAS PEQUENAS EMPRESAS
}

Talita Bernardi Goettems ${ }^{1}$

Denize Grzybovski ${ }^{1}$

${ }^{1}$ Universidade de Passo Fundo 


\section{CONSTITUIÇÃO DE COMUNIDADES DE PRÁTICA NAS PEQUENAS EMPRESAS}

\section{Resumo}

O presente ensaio teórico tem como objetivo problematizar teoricamente as questões inerentes à constituição de comunidades de práticas nas pequenas empresas, considerando o contexto brasileiro, refletindo sobre suas possibilidades e limites. São analisados os pressupostos teóricos das comunidades de prática e as especificidades da gestão das pequenas empresas brasileiras. Os argumentos residem na gestão do conhecimento organizacional, cujo campo teórico pressupõe as organizações fracamente estruturadas como sendo as que tem maior potencial de inovação. Outro argumento encontra-se nas comunidades de prática, cuja estrutura precisa ser informal para existir, mesmo quando o espaço de sua constituição seja uma organização formal. Conclui-se que, em pequenas empresas, a proximidade física dos seus membros e os espaços organizacionais fracamente estruturados são condições que possibilitam a emergência de comunidades de práticas e que, no contexto brasileiro tais possibilidades são maiores, tendo em vista as questões antropológicas e sociológicas do povo empreendedor brasileiro.

Palavras-chave: Comunidades de prática. Gestão de Pequena empresa. Conhecimento Organizacional. Aprendizagem Organizacional. 


\section{Provocações iniciais}

A noção de comunidades de prática entrelaça seus dois conceitos constituintes (comunidade e prática) com o propósito de criar, expandir e partilhar conhecimentos, que pode evoluir organizadamente (SOUZA-SILVA, 2007). Em comunidades de prática não ocorre a simples transferência de conhecimento que isola o conhecimento da prática, como descrevem Lave e Wenger (1991). Trata-se de uma estrutura organizacional que recoloca o conhecimento num contexto que abriga significado (BROWN; COLLINS; DUGUID 1989; PEA, 1990). Isto reforça a ideia que o conceito de comunidade de prática não nasceu na tradição da teoria dos sistemas, mas foi inspirada nos fundamentos teóricos da Antropologia e da Teoria Social, os quais encontram-se descritos em Bourdieu (1977), Foucault (1980), Giddens (1984) e Lave (1988).

A noção, e também estrutura organizacional de comunidade de práticas foi descrita por Lave e Wenger (1991) no início da década de 1990, fruto de estudos sobre a aprendizagem situada, que consistem em grupos informais de pessoas que compartilham conhecimentos e se entusiasmam pela prática organizativa. Nelas, afirma Wenger (1998), as pessoas disseminam conhecimento especializado e sentem paixão pelo tema de domínio da comunidade, por isso representam uma das formas sociais que estruturam a colaboração no local de trabalho (LAVE; WENGER, 2006) pela participação efetiva das pessoas.

O conceito não existe por si só, faz parte de um quadro conceitual mais amplo para pensar sobre aprender em suas dimensões sociais. É uma perspectiva que localiza o aprendizado, não no cérebro das pessoas ou fora dele, mas na relação entre a pessoa e o mundo, na qual o social e o indivíduo constituem um ao outro (WENGER, 2010). Dessa forma, aprendizes podem de uma maneira ou de outra construir as circunstâncias físicas, histórias e de relações sociais de forma que o conteúdo apreendido esteja profundamente conectado às condições nas quais é aprendido.

Com base no exposto é possível pressupor que estruturas organizacionais flexíveis são propícias para constituir comunidades de prática, contudo gera um questionamento: as estruturas organizacionais predominantes nas pequenas empresas brasileiras, considerando as questões antropológicas e sociológicas de contexto, representam as condições ideias para constituir comunidades de práticas? Em caso afirmativo, quais seriam seus limites?

Problematizar teoricamente as questões inerentes à constituição de comunidades de práticas nas pequenas empresas, considerando o contexto brasileiro, bem como as possibilidades e os limites é objeto deste ensaio teórico. O estudo se apoia no entendimento de Oliveira et al. (2014) sobre a gestão do conhecimento em empresas de menor porte (micro, pequenas e médias), quem afirma que é favorecida pelo horizontalização da estrutura organizacional, cujas características são de organização fracamente estruturada, as quais facilitam o compartilhamento de conhecimentos entre seus membros, mas também dificulta a ação da concorrência em imitar o que é produzido na informalidade do espaço físico de convivência dos mesmos.

A justificativa está no fato de que o conhecimento está enraizado nos praticantes, não na organização (SILVA, 2014), contudo há uma variedade de formas que uma comunidade de prática pode apresentar, seja pelo grau de informalidade e/ou nível de autonomia, não podendo ser confundida com outras configurações sociais (SOUZA-SILVA, 2007).
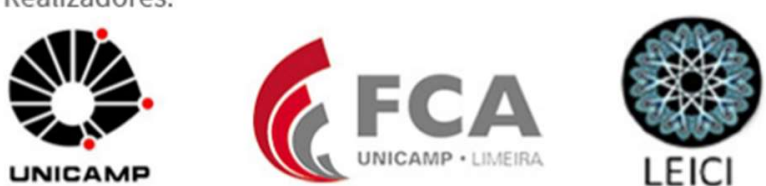
No entendimento de Gnecco Junior et al. (2012), comunidades de prática é uma técnica de gestão do conhecimento definida por sua origem informal tendo por base o interesse de um grupo que troca informações e contribuições, seja por interesse ou iniciativa e a condição de sua existência é um empreendimento comum entre seus membros.

\section{Para além da transferência de conhecimento}

No entendimento de Lave e Wenger (1991), as comunidades de prática possibilitam a "participação periférica legítima". Uma comunidade de prática é um grupo de pessoas que se aglutinam entre si para se desenvolverem em um domínio do conhecimento, vinculado a uma prática específica. Assim, as pessoas colaboram reflexivamente, partilham experiências, conhecimentos e soluções para problemas ligados às suas práticas (WENGER, 1998).

Nas comunidades de prática, as relações são criadas em atividades e as atividades se concretizam por meio das relações sociais e experiências daqueles que as formam para que o conhecimento e as habilidades se tornem parte da identidade individual e encontrem sua colocação na comunidade. Ao mesmo tempo, a dimensão da comunidade é uma condição essencial para a existência do conhecimento prático (GHERARDI; NICOLINI, 2001). O conceito de comunidade de prática representa uma estrutura social propícia não só para a disseminação do conhecimento, mas para o processo de inovação, pois ativa a colaboração reflexiva (SOUZA-SILVA, 2007).

O conceito de comunidades de prática emerge como algo distinto daqueles conceitos tradicionais, por exemplo, de grupo ou equipe de trabalho, que são formas organizacionais que produzem resultados formais. O surgimento do conceito sempre esteve atrelado aos aspectos informais dos relacionamentos para produzir conhecimentos não canônicos. Além disso, pode-se observar que a evolução no conceito de comunidades de prática possibilitou novas formas de utilizá-lo. Apesar da falta de unicidade evidente no conceito de comunidades de prática, a existência desse conceito coloca limites em suas apropriações. E tais limites estão condicionados, por exemplo, à forma como o grupo é visto (informal e/ou por interesses dos participantes) e às necessidades diversas de aprendizagem (informal, situada, colaborativa e na prática), as quais induzem à formação das comunidades de prática (MENDES; URBINA, 2015).

Uma comunidade de prática pode ser vista como um sistema de aprendizagem social. Decorrente da aprendizagem, exibe muitas características dos sistemas: estrutura, relações complexas, auto-organização, limites dinâmicos entre outros. Considera-se uma unidade social simples que tenha as características de um sistema de aprendizagem social (WENGER, 2010). As comunidades de prática não estão isoladas de um determinado grupo, organização/comunidade maior. Elas são parte de uma sociedade mais ampla, de sistemas sociais que envolvem outras comunidades/estruturas, como projetos, movimentos sociais, empresas cujo objetivo é gerar novos conhecimentos para seus membros de maneira informal, cujas consequências indiretas é o aprimoramento de práticas de trabalho e inovação contínua.

Por isso uma comunidade de prática não é simplesmente uma equipe de trabalho, porque ela se define pelo domínio de interesse e não por um trabalho a realizar. Também não é uma rede informal, porque, além de interesses em comum, ela tem uma identidade própria (MOURA, 2009).

Organizadores:
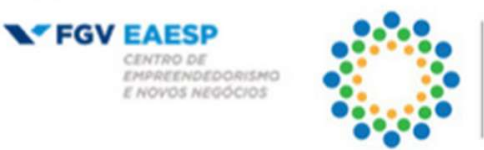

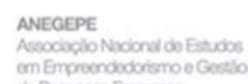
do Pequeras Erprosess

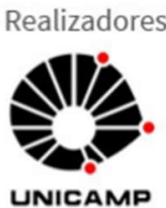


Aprendizagem, do ponto de vista da participação periférica legítima (LPP), essencialmente envolve um insider. Aprendizes não recebem ou mesmo constroem conhecimento abstrato, objetivo e individual; no entanto, eles aprendem o funcionamento dentro de uma comunidade, seja uma comunidade de físicos nucleares, fabricantes de armários, estudantes de ensino médio, sociedade de esquina de rua e etc... Eles assumem o ponto de vista subjetivo daquela comunidade em particular e aprendem a falar seu idioma; são aculturados (BROWN; COLLINS; DUGUID, 1989). Aprendizes não estão adquirindo conhecimento explícito, mas a habilidade incorporada no comportamento como membros da comunidade.

A aprendizagem é um processo intelectual, emocional, onde a comunidade de prática constitui-se em ideal tecido social de partilha e disseminação de conhecimento, pois combina na sua própria dinâmica, aspectos cognitivos e emocionais tão necessários para que a aprendizagem aconteça em sua integridade. Assim, na medida que os trabalhadores se engajam em comunidades de prática se intensifica a partilha de conhecimento organizacional (SOUZA-SILVA, 2007). Para tanto as comunidades de práticas criam benefícios tanto no nível individual, para o praticante, quanto no nível organizacional (para a empresa), como está demonstrado no Quadro 1.

Quadro 1 - Benefícios das comunidades de práticas nos níveis organizacional e individual

\begin{tabular}{|c|c|c|}
\hline \multirow{2}{*}{$\begin{array}{l}\text { Nível de } \\
\text { análise }\end{array}$} & \multicolumn{2}{|c|}{ Benefícios produzidos pelas comunidades de práticas } \\
\hline & Curto prazo & Longo prazo \\
\hline Organizacional & $\begin{array}{l}\text { - Fórum para incrementar a qualidade } \\
\text { do processo decisório; } \\
\text { - Promoção de maior sinergia } \\
\text { interdepartamental; } \\
\text { - Ideal configuração social para a } \\
\text { disseminação de conhecimento. }\end{array}$ & $\begin{array}{l}\text { - Retenção de talentos profissionais; } \\
\text { - Capacidade de desenvolvimento de novas } \\
\text { estratégias organizacionais; } \\
\text { - Habilidade para prever desenvolvimentos } \\
\text { tecnológicos e mudanças ambientais. }\end{array}$ \\
\hline $\begin{array}{l}\text { Individual } \\
\text { (praticantes) }\end{array}$ & $\begin{array}{l}\text { - Acesso à competência legitimada de } \\
\text { comunidade; } \\
\text { - Apoio técnico e emocional nos } \\
\text { desafios profissionais; } \\
\text { - Senso de pertencimento. }\end{array}$ & $\begin{array}{l}\text { - Fórum para expandir talentos; } \\
\text { - Rede para se manter atualizado com novos } \\
\text { conhecimentos e tecnologias; } \\
\text { - Reputação e identidade profissional } \\
\text { incrementada. }\end{array}$ \\
\hline
\end{tabular}

Fonte: Adaptado de Souza- Silva (2007).

Comunidades de prática são definidas como um conjunto de relações entre pessoas que têm algo em comum (LAVE; WENGER, 2006). Também é um lugar para a aprendizagem nas organizações (WENGER, 1998), pois a aprendizagem é situada e ocorre por práticas progressivas, sendo que relacionamentos e interações constantes nos locais de trabalho reforçam uma comunidade e contribuem para que a informalidade característica do espaço organizacional seja fértil para criação, armazenagem e gestão de conhecimento organizacional.

Na perspectiva do processo social, o aprendizado já não é equiparado à apropriação ou aquisição de conhecimentos. É visto como o desenvolvimento de identidades situadas com base na participação em uma comunidade de prática (LAVE; WENGER, 1991). Segundo a
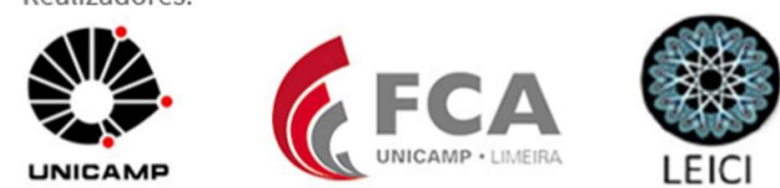
afirmativa de Gherardi (1991) aprendizado não é concebido como uma forma de conhecer o mundo, mas como uma maneira de estar no mundo.

O conhecimento se faz presente nas pequenas empresas pelas suas especificidades, se encontra enraizado nas pessoas, porém se perde, não tem significado para o indivíduo, está estruturado. Assim, a partir desse estudo apresenta-se uma nova perspectiva, com a construção da sua própria compreensão, por meio das comunidades de práticas.

\section{Comunidades de práticas na gestão das pequenas empresas}

As pequenas empresas, geralmente caracterizadas pelo número de trabalhadores (JAMIL; MOHAMED, 2011; SEBRAE, 2016), são descritas como um estrato da população de empresas de um país que oferece produtos/serviços alternativos ao mercado ou que não são de interesse das grandes empresas. Ainda, apresentam configuração societária a típica, pois na maioria das vezes é formada pelo fundador e sua família.

Leone (1999) afirma que não é possível definir ou categorizar as pequenas empresas com apenas um único conceito, pois elas não têm o mesmo comportamento econômico e social, mesmo pertencentes a um único conjunto de dados. Porém, são importantes para a pesquisa na medida em que, ao conhecer suas dinâmicas, estruturas, modelos e ferramentas de gestão, contribui na compreensão das suas especificidades. Além disso, Leone (1999) divide a especificidades das pequenas e médias empresas em especificidades organizacionais, sendo pobreza de recursos, gestão centralizadora, situação extraorganizacional incontrolável, fraca maturidade organizacional, fraqueza das partes no mercado, estrutura simples e leve, ausência de um planejamento formal de trabalho, fraca especialização, estratégia intuitiva e pouco formalizada. Quanto às especificidades decisionais, a referida autora afirma que o processo decisório é baseado na intuição do empresário, na sua experiência profissional e no horizonte de curto prazo. Tal comportamento organizacional é explicado pela inexistência de dados quantitativos sobre o desempenho das diferentes áreas funcionais, pois o empresário tem alto grau de autonomia decisória, utiliza a lógica econômica e as necessidades da família proprietária para orientar sua decisão.

No que se refere às especificidades individuais estão à onipotência do proprietário dirigente, identidade entre pessoa física e jurídica, dependência perante certos trabalhadores, influência pessoal do proprietário-dirigente, simbiose entre patrimônio social e pessoal, propriedade dos capitais e propensão a riscos calculados (LEONE, 1999). Dessa forma, pode se considerar um ambiente propicio para o desenvolvimento das comunidades de práticas, para que o conhecimento dos trabalhadores se torna fator importante e não se perde, trazendo benefícios para esse âmbito organizacional.

As pequenas empresas possuem algumas particularidades que influenciam sua atuação no mercado e demandam um processo diferenciado de gestão de forma a contemplar os aspectos comportamentais, estruturais e contextuais. Terence e Escrivão Filho (2001) e Grzybovski (2002) afirmam que a gestão das pequenas empresas reflete valores, ambições, ideologias, visões e comportamentos do proprietário e/ou da família proprietária.

As particularidades estruturais são as mais citadas na literatura e referem-se: a informalidade das relações na pequena empresa, estrutura organizacional não formalizada e reduzida, a falta de recursos e pessoal qualificado, a inadequação ou não utilização das
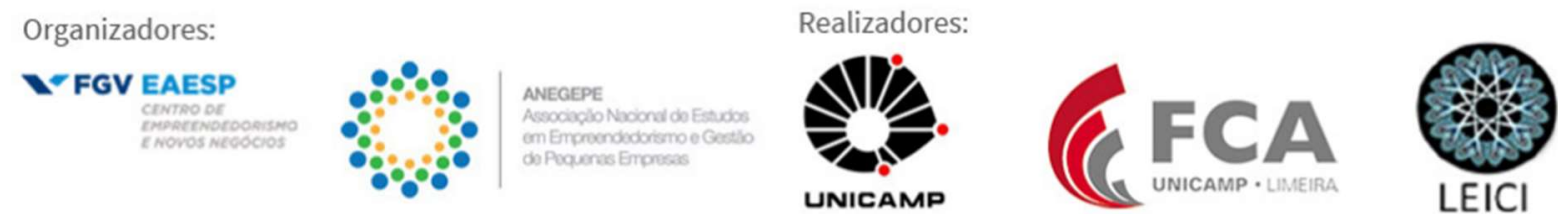
técnicas gerenciais e o planejamento. Por fim as particularidades contextuais referem-se ao ambiente externo da pequena empresa e não são controláveis pela ação individual dos dirigentes empresariais entre elas estão: a carência de informação sobre a evolução do mercado e de seus produtos, a dificuldade de acesso a processos de treinamento gerencial e a fontes de financiamento, entre outras (TERENCE; ESCRIVÃO FILHO, 2001).

A literatura anglo-saxônica segue um paradigma orientador da pesquisa em pequenas empresas orientado pela tese da especificidade gerencial. Nesse paradigma, o pequeno negócio é considerado uma entidade específica, com problemas administrativos distintos dos da grande empresa, com destaque para: estrutura administrativa centralizada; estratégias intuitivas e de curto prazo; baixa especialização; simplicidade e informalidade do sistema de informação e atuação em mercados locais. Essa concepção tornou-se fundamental para acentuar a contraposição entre pequena e grande empresa e para identificar o papel das pequenas empresas no sistema econômico (SOUZA; MAZZALI, 2008). No entanto, as pequenas empresas são mais suscetíveis a especificidades de contexto, o que exige uma análise mais detalhada na perspectiva antropológica envolvendo o país, a região, o lugar.

Pecanha e Oliveira (2015) afirmam que as empresas familiares brasileiras apresentam traços da cultura nacional amplamente descritos pelos antropólogos Giberto Freyre, Roberto Da Mata e tantos outros. Especificamente Pecanha e Oliveira (2015) descrevem os seguintes traços característicos na gestão das pequenas empresas familiares:

a) Jeitinho - Visa à harmonização das regras e determinações universais da vida com as necessidades diárias do cidadão, buscando a realização de objetivos a respeito de determinações legais contrárias e podendo ser visto como uma forma de sobreviver ao cotidiano, um recurso de resistência cultural.

b) Desigualdade de poder e hierarquia - percebida quando muitos indivíduos se julgam com direitos especiais que os eximem de sujeitar-se à lei de caráter generalizante. A expressão popular "você sabe com quem está falando?

c) Flexibilidade - Permeia os comportamentos nas organizações e traduz-se a capacidade de adaptação e criatividade das pessoas.

d) Personalismo - Indica o alto grau de confiança depositado na rede de amigos e familiares para resolução de problemas ou obter privilégios.

e) Formalismo - comportamentos que buscam por um lado a reduzir o risco, da ambiguidade e da incerteza e, por outro, o aumento de previsibilidade e controle sobre as ações e comportamentos humanos.

Tais características de contexto interferem na gestão da pequena empresa e precisam ser consideradas na análise das comunidades de prática no seu interior. Se, por um lado, a pequena empresa apresenta limitações de recursos face à grande empresa, por outro ela tem vantagens, sendo uma delas o ambiente informal, que pode favorecer a criatividade. A proximidade é uma vantagem importante que a pequena empresa conta para produzir e disseminar o conhecimento, bem como o tamanho reduzido da equipe, que pode favorecer o domínio de atividades, sendo que muitas vezes há um rodizio, ocorrendo às vezes até mesmo acúmulo de funções. Outra característica refere-se ao papel central do empreendedor nas atividades e na definição de estratégias para o pequeno negócio (MACHADO, 2016).

No entendimento de Belmonte et al. (2009), o conhecimento gerado pela informalidade nas pequenas empresas é reconhecido como um diferencial. Porém, muitas
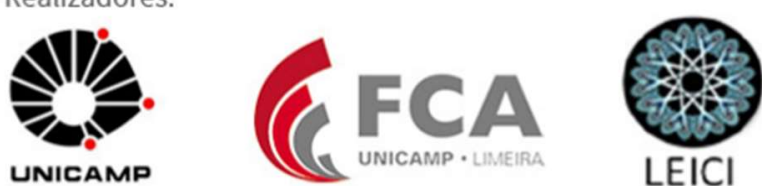
vezes, a informação se encontra inacessível, desorganizada e dispersa nos diferentes espaços organizacionais. Assim, reconhecer tais espaços como de comunidades de prática seria reconhece-los como geradores de conhecimentos organizacionais.

\section{Reflexões finais}

Uma comunidade de prática pode apresentar várias formas, o importante é o surgimento na informalmente, onde os participantes possuam significativos níveis de autonomia, pois se a organização tentar estruturar (formalizando) a determinada comunidade de prática deixará de existir.

Os teóricos da aprendizagem rejeitam o modelo de transferência de conhecimento que isola o conhecimento da prática e desenvolveram uma visão de aprendizagem como construção social. Nesse sentido, uma das possibilidades das comunidades de prática em pequenas empresas é tornar o conhecimento produzido por ela um fator de produção para a criação de inovação continuamente. O limite, no entanto, é a capacidade deste estrato de empresas reconhecer um conhecimento produzido pela comunidade, como de valor e a capacidade de manter a informalidade da comunidade, sem interferir na sua dinâmica de estrutura e de produção.

Em comunidades de prática, o conhecimento não se perde, mas é preciso ter cuidado para reconhecer sua existência e sua origem, a qual reside na informalidade da comunidade, sem tentar estruturar no espaço formal, seja denominando-a "grupo de trabalho" ou apenas de projeto. Nesse sentido se reconhece nas características antropologias e sociológicas do povo e dos empreendedores brasileiros um potencial para a emergência de comunidades de práticas em pequenas empresas, sendo elas: informalidade nas relações de trabalho, horizontalização dos cargos, flexibilidade e coletividade nos processos decisórios.

Ao considerar que as especificidades requeridas na gestão das pequenas empresas brasileiras apresentam a diversidade dos povos que formaram a identidade do brasileiro e o sentido do Brasil (RIBEIRO, 1997), emergem novas questões para orientar pesquisas futuras sobre o tema, as quais encontram-se alinhadas ao pensamento original de Guerreiro Ramos (1981; 1983). Será que gerenciar pequenas empresas no Brasil é formatar processos, estruturar o conhecimento, ou seja, seguir as orientações da mainstream? O debate apresentado neste ensaio teórico revela que não, porém há distância de um ambiente organizacional positivo para criação de conhecimentos. É preciso estabelecer como propósito, nas pesquisas sobre a gestão das pequenas empresas brasileiras, naturalizar a administração no contexto nacional distanciando-se das orientações anglo-saxônicas e perseguir um ideal de emancipação. Assim é possível alinhar às orientações de Davel e Alcadipani (2002) sobre o que é ser crítico e tentar aproximar as questões específicas sobre a gestão de pequenas empresas brasileiras e a gestão do conhecimento organizacional para pensar a capacidade de pequenas empresas familiares brasileiras constituírem comunidades de práticas.

Por fim, ao abrir espaço para aproximar os debates desenvolvidos no campo de estudos da aprendizagem organizacional, no qual são rejeitados modelos, práticas e políticas que separam o conhecimento da prática, será possível propor uma visão de construção social da realidade organizacional específica das pequenas empresas e adequadas/adaptadas ao contexto brasileiro. Em pequenas empresas brasileiras, a proximidade física dos seus
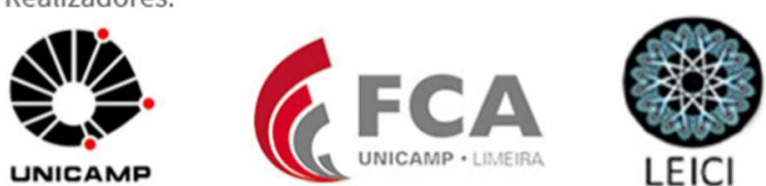
membros e os espaços organizacionais fracamente estruturados são consideradas potencialidades para a constituição de comunidades de práticas e uma possibilidade em pesquisadores organizacionais brasileiros continuarem a perseguir a renovação da teoria da administração iniciada por Alberto Guerreiro Ramos.

\section{Referências}

BELMONTE, D. L. et al. A gestão do conhecimento nas pequenas e médias empresas brasileiras. Publication UEPG: Ciências Sociais Aplicadas, v. 13, n. 2, 2009.

BOURDIEU, P. Outline of a Theory of Practice. Cambridge: Cambridge University, 1977.

BROWN, J. S.; COLLINS, A.; DUGUID, P. Situated cognition and the culture of learning. Educational Researcher, v. 18, n. 1, p. 32-42, 1989.

DAVEL, E., ALCADIPANI, R. Estudos críticos em administração: reflexões e constatações sobre produção brasileira. In: ENCONTRO DE ESTUDOS ORGANIZACIONAIS, 2, 2002, Recife. In: Anais do... Observatório da Realidade Organizacional: PROPAD/UFPE: ANPAD, 2002.

FOUCAULT, M. Power/Knowledge: selected interviews and writings. New York: Pantheon, 1980.

GIDDENS, A. The constitution of society: outline of the theory of structuration. University of California, 1984.

GHERARDI, S; NICOLINI, D. The organizational learning of safety in communities of practices. Journal of Management Inquiry, v. 9, n. 1, p. 7-18, 2001.

GNECCO JUNIOR, L.; SANTANA J. Q., DALMAU M. B. L.; SANTOS, N.; RADOS G. J. V. Métodos e técnicas de gestão do conhecimento: comunidades de prática. Reuna, Belo Horizonte. v. 17, n. 2, p. 59-80, Abr/Jun. 2012.

GRZYBOVSKI, D. O administrador na empresa familiar: uma abordagem comportamental. Passo Fundo: UPF, 2002.

JAMIL, C. M.; MOHAMED, R. Performance measurement system (PMS) in small medium enterprises (SMES): a practical modified framework. World Journal of Social Sciences, v. 1, n. 3, p. 200-212, July, 2011.

LEONE, N. M. C. P. G. As especificidades das pequenas e médias empresas. Revista de Administração, v. 34, n. 2, p. 91-94, 1999.

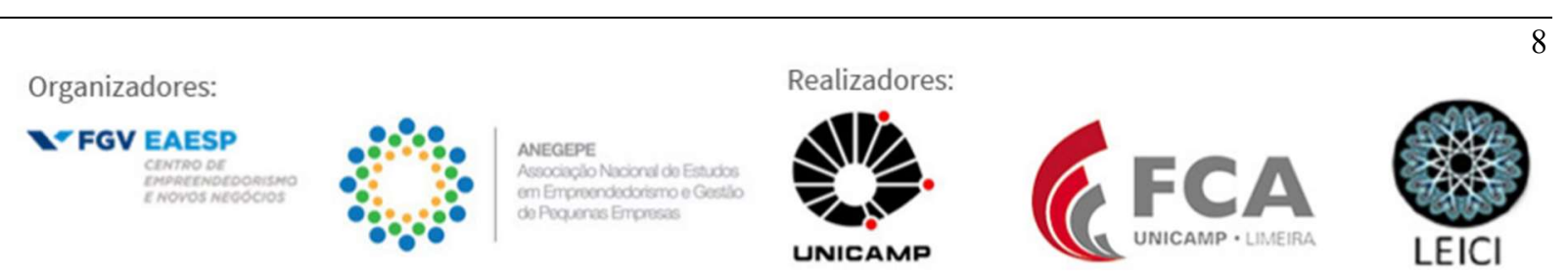


LAVE, J. Cognition in practice: mind, mathematics, and culture in everyday life. Cambridge University, 1988.

LAVE, J.; WENGER, E. Situated learning: legitimate peripheral participation. Cambridge: Cambridge University, 1991.

MACHADO, H. V. Especificidades do conhecimento em pequenas empresas e perspectivas de pesquisas. In: ENCONTRO DE ESTUDOS SOBRE EMPREENDEDORISMO E GESTÃO DE PEQUENAS EMPRESAS, 9, 2016, Passo Fundo. Anais do... Passo Fundo: Anegepe, 2016.

MENDES, L.; URBINA, L.M.S. Análise sobre a produção acadêmica brasileira em comunidades de prática. Revista de Administração Contemporânea. v. 19, n. 4, p. 305-327, Out, 2015.

MOURA, G. M. Somos uma comunidade de prática? Revista de Administração Pública, v.43, n. 2, p.323-346, Mar./Abr. 2009.

OLIVEIRA, M. et al. Gestão do conhecimento em micro, pequenas e médias empresas.

Interfaces, v. 1, n. 4, Porto Alegre, 2014.

PEA, R. D. Distributed cognition. Palo Alto: Institute for Research on Learning, 1990.

PECANHA, A. R. O.; OLIVEIRA, S. B. de. Empresa familiar, sim! Mas qual o problema se a gestão é profissional? Revista de Gestão e Tecnologia, v. 5, n. 3, p. 126-135, jul/set, 2015.

RAMOS, A. G. A nova ciência das organizações: uma nova ciência das organizações. Rio de Janeiro: FGV, 1981.

RAMOS, A. G. Administração e contexto brasileiro. Rio de Janeiro: FGV, 1983.

RIBEIRO, D. O povo brasileiro: a formação e o sentido do Brasil. 2.ed. São Paulo: Companhia das Letras, 1997.

SEBRAE. Serviço Brasileiro de Apoio às Micro e Pequenas Empresas. Sobrevivência das empresas no Brasil. Brasília: Sebrae, 2016.

SILVA, G. A. S.; RAMOS, C. O.; BONANZA, V.; SILVA, L.; BEZERRA, M. S.; CRISTINA, L. C. C. Como se caracteriza a relação entre a gestão de pessoas no CSC (Centro de Serviços Compartilhados) e as políticas e estratégias da organização. Revista Eletrônica Gestão e Serviços. v.5, n.1, p. 743-765, Jan/Jun, 2014 
SOUZA, M. C. A. F.; MAZZALI, L. Conceito e espaço da pequena empresa na estrutura industrial: heterogeneidade e formas de inserção. Gestão \&Produção, v. 15, n. 3, Set/Dez. 2008.

SOUZA-SILVA, J. C. Aprendizagem organizacional: desafios e perspectivas ao desenvolvimento de comunidades de prática. Salvador: Conhecimento Superior, 2007.

TERENCE, A. C. F.; ESCRIVÃO FILHO, E. Particularidades das pequenas empresas no planejamento estratégico: a elaboração de um roteiro prático. In: ENCONTRO DE ESTUDOS SOBRE EMPREENDEDORISMO E GESTÃO DE PEQUENAS EMPRESAS, 2, 2001, Londrina. Anais do... Londrina: Anegepe, 2001.

WENGER, E. Communities of practice: learning, meaning, and identity. Cambridge: Cambridge University, 1998.

WENGER, E. Communities of practice and social learning systems: the career of a concept. In: BLACKMORE, C (ed.). Social learning systems and communities of practice. London: Springer Verlag and the Open University, 2010. Capítulo 11, p. 179-198. 